\title{
Non-invasive glucose monitoring devices: A review
}

\author{
Muhammad Farhan Affendi Mohamad Yunos, Anis Nurashikin Nordin \\ Department of Electrical and Computer Engineering, Kulliyyah of Engineering, IIUM, Malaysia
}

\section{Article Info}

Article history:

Received Feb 6, 2020

Revised Apr 10, 2020

Accepted May 3, 2020

\section{Keywords:}

Diabetes

Glucometer

Non-invasive biosensor

RF sensor

Wearables

\begin{abstract}
Diabetes is a growing chronic disease that affect millions of people in the world. Regular monitoring of blood glucose levels in patients is necessary to keep the disease under control. Current methods of blood glucose monitoring devices are typically invasive, causing discomfort to the patients. Non-invasive glucose monitoring devices are a possible game changer for diabetic patients as it reduces discomfort and provides continuous monitoring. This manuscript presents a review of non-invasive glucose biosensors with particular focus on leading technologies available in the market, such as microwave sensing, near-infrared spectroscopy, iontophoresis, and optical methods. This paper intends to describe non-invasive blood glucose monitoring methods using various biological fluids (sweat, saliva, interstitial fluid, urine), highlighting the advantages and drawbacks in latest device development. This review also discusses future trends of glucose detection devices and how it will improve patients' quality of life.
\end{abstract}

This is an open access article under the CC BY-SA license.

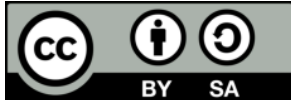

\section{Corresponding Author:}

Anis Nurashikin binti Nordin,

Department of Electrical and Computer Engineering, Kulliyah of Engineering,

International Islamic University Malaysia,

Jalan Gombak, 53100, Selangor, Malaysia.

Email: anisnn@iium.edu.my

\section{INTRODUCTION}

Diabetes Mellitus is a major health concern in the society today both locally and worldwide [1]. According to National Diabetes Institute Malaysia, 3.6 million Malaysians suffer from diabetes in 2019. This is the highest rate of incidence in Asia and one of the highest in the world. Diabetes has been linked with other health problems, including heart disease and stroke, kidney disease, nerve damage and vision loss due to fluctuating blood sugar levels. Such complications can result in retinopathy leading to blindness, can lead to amputation of feet and legs, neuropathies (nerve disorder), cardiovascular diseases or even worst; death [2]. Diabetes Mellitus can be categorized into two major types, Type 1 and Type 2. Type 1 occurs when insulin cannot be produced in the body to control the level of blood glucose. Type 2 which is common, occurs when not enough insulin is produced or when the insulin in the body is not working efficiently [3]. Pre-diabetes is a health condition where patients have a higher level of blood glucose than normal but not high enough to be classified as diabetes.

There are several signs and symptoms of pre-diabetes includes weight loss, a frequent desire to urinate, excessive thirst and blurred vision. Pre-diabetes must be taken seriously as it increases the chance of developing Type 2 diabetes. Both Type 1 and Type 2 diabetes cannot be cured, the best treatment method for patients is to have regular measurements and monitoring of the blood glucose levels to keep the disease under control. Unfortunately, these blood sugar levels have to be monitored for the rest of their lives as diabetes patients are prone to have either hypoglycaemia (low blood sugar) or hyperglycaemia (high blood sugar). Long-term monitoring of the blood sugar levels in patients has been reported to prolong their life expectancies [4]. Blood glucose monitoring can be coupled with monitoring of other lifestyle changes in 
the patient such as diet and physical activity to optimize strategies for patient treatment [5]. This paper presents an overview of non-invasive glucose sensors for diabetes monitoring. Biological fluids that can be used other than blood for continous glucose monitoring will be discussed. Latest technology in commercial remote glucose have been reviewed and its advantages and disadvantages are compared. Section 3 presents different sensing techniques for glucose detection and the latest available commercial devices. Finally, the conclusions and the future trends are presented.

\section{GLUCOSE SENSING FOR DIABETES MONITORING}

\subsection{Overview}

Due to the long term requirements of blood glucose monitoring in diabetic patients, regular self-testing and patient compliance to medication is crucial to prevent diabetes complications. Currently, diabetes patients can test the blood glucose at home with a portable electronic device (glucose meter) that measures sugar level in a small drop of blood. Continuous blood glucose monitoring is advantageous for diabetic patients as it can provide feedback on the effectiveness of the prescribed treatment. Diabetes management is a multi-pronged and some doctors may prescribe controlled diet, exercise in addition to the medication. Having regular measurements of blood sugar levels of the patients provide important information to the doctors on how well their patients are responding to the treatment plan. Table 1 shows the different categories of blood glucose concentration for normal, high risk and diabetic adults. The blood glucose concentration in the body fluctuates during the day and normally increases after every meal. Figure 1 shows the variation in blood glucose (red), insulin (blue) levels throughout the day. It can be seen that a sugar-rich meal will cause the blood sugar level to spike, rising to levels above $6 \mathrm{mg} / \mathrm{dL}$. Persistent elevation of blood glucose values may lead to glucose toxicity, causing cell dysfunction and complications of diabetes [6].

Table 1. Ranges for blood glucose concentration in (mg/dL) [7]

\begin{tabular}{cc}
\hline Categories & Fasting blood glucose concentration) \\
\hline Normal & $<100 \mathrm{mg} / \mathrm{dL}$ \\
High Risk & $100-125 \mathrm{mg} / \mathrm{dL}$ \\
Diabetes & $>=126 \mathrm{mg} / \mathrm{dL}$ \\
\hline
\end{tabular}

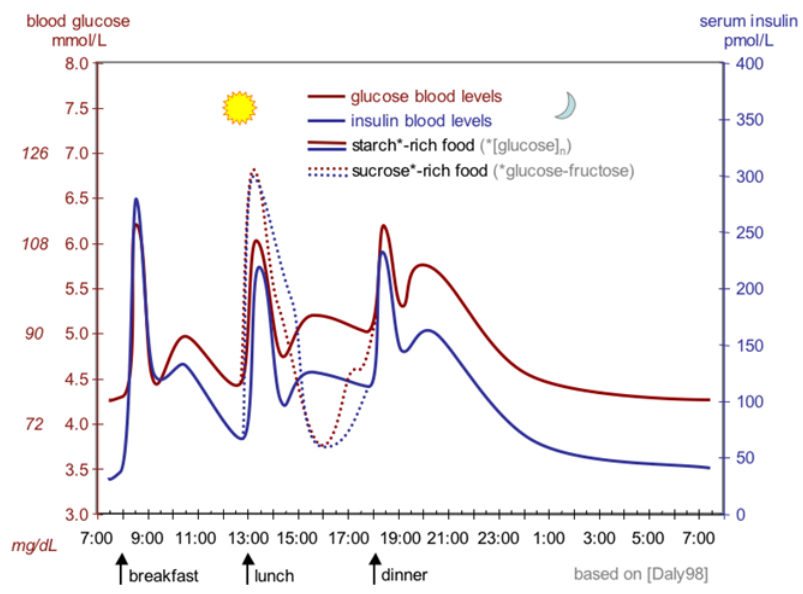

Figure 1. Blood sugar fluctuations in adults. Glucose blood levels (red), insulin or sugar lowering hormone levels (blue) when consuming starch rich food (solid lines), sugar rich food (dotted lines) [8]

\subsection{Body fluids suitable for glucose monitoring}

For decades, blood has been known as the common body fluid used as sample for measurements of various test. Blood glucose measurements are usually made from the fingertips, also known as capillary blood. The glucose levels in the capillaries have been shown to correlate well with systemic arterial blood glucose levels. When the patient is stable, identical glucose levels can be observed from fingertip and other sites such as forearm etc. There are also other accessible body fluids suitable for the tests such as interstitial fluid, sweat, ocular fluid, urine or saliva for the continuous monitoring of non-invasive methods. Interstitial 
fluid is a thin layer fluid that surrounds our cells in the body. It consists mostly of water together with sugars, salts, fatty acids, amino acids, coenzymes, hormones, white blood cells, neurotransmitters and cell by-products. Due to its contents, it provides an alternative method of sampling glucose levels in the body other than blood although glucose levels in interstitial fluids is different from those and correlation calculations are required. There is also a delay in transmission of glucose levels from the blood to the interstitial fluids. Interstitial fluids can also be used to detect inherited metabolic disease, drug efficacy and organ failure. These fluids are easily accessible from the subcutaneous tissue just underneath the skin and newer glucometers have incorporated sensors in the skin to continuously measure glucose levels in the body. Due to its proximity to the skin, minimally invasive microneedles can be used to collect interstitial fluids for sensing purposes. These tiny electrodes can be attached on the skin using an adhesive layer for up to 14 days, allowing continuous monitoring of sugar levels in the body.

Urine is another type of body fluid that can be used for diabetes diagnostics and is easily accessible and is non-invasive. Urine is composed of several metabolites that includes glucose, nitrates, dissolved salts and protein. The presence of metabolites causes the $\mathrm{pH}$ of urine fluctuate between acidic ( $\mathrm{pH} 4.8)$ to basic $(\mathrm{pH} 8)$. Glucose can be found in urine when the amount is high as it will be excreted from blood to urine and is detectable using the glucose test. Unfortunately, urine cannot be used for continuous glucose-monitoring due to its intermittent nature. Sweat, is known as thermoregulatory body fluid as it helps to regulate the temperature of the body. It is the most accessible body fluid which is generated by the sweat glands to help stabilize the temperature of the body. The eccrine sweat glands are distributed throughout the human body but excretes most sweat in feet, hand, underarm and lower back. Sweat is slightly acidic (pH 5.5-6.5) and is composed mainly of water, electrolytes, urea with smaller amounts of glucose, antigens, antibodies and cytokines [9].

The glucose levels in sweat can be correlated with blood glucose levels (5\%-20\%) although it lags by about 8 minutes the levels in blood [10]. Saliva is considered as complex fluid as it contains many analytes excreted from blood which able to affect a person's hormonal, emotional, nutritional and metabolic state. Saliva can act as a sample for non-invasive glucose sensing. The range of glucose levels in saliva for healthy person ranges from 0.23 to $0.38 \mathrm{mM}$. Meanwhile, the level of glucose for diabetic patients in saliva ranges from 0.55 to $1.77 \mathrm{mM}$. More research needs to be done to have an accurate relationship between glucose levels in blood and saliva, before it can be used with therapeutic intervention [11]. Despite this, saliva still offers various advantages for diagnostics as it is a non-invasive method. A lot of research in this aspect has been done and there are many emerging technologies reported for continuous and non-invasive glucose detection in saliva using daily dental devices such as mouth guards, dentures and dental tattoos [11]. Table 2 summarizes the different body fluids that can be used for glucose detection and their glucose concentrations.

Table 2. Summary of glucose concentrations values measured in physiological fluids of healthy and diabetic patients [11]

\begin{tabular}{ccc}
\hline Physiological Fluid & Glucose Concentration for Healthy Patients & Glucose Concentration for Diabetic Patients' \\
\hline Blood & $4.9-6.9 \mathrm{mM}$ & $8-40 \mathrm{mM}$ \\
Interstitial fluid & $3.9-6.6 \mathrm{mM}$ & $7.99-22.2 \mathrm{mM}$ \\
Urine & $2.78-5.55 \mathrm{mM}$ & $5.55 \mathrm{mM}$ \\
Sweat & $0.06-0.11 \mathrm{mM}$ & $0.3-1 \mathrm{mM}$ \\
Ocular fluid & $0.05-0.5 \mathrm{mM}$ & $0.5-5 \mathrm{mM}$ \\
Saliva & $0.23-0.38 \mathrm{mM}$ & $0.55-1.77 \mathrm{mM}$ \\
\hline
\end{tabular}

\section{GLUCOSE DETECTION: SENSING TECHNIQUES}

\subsection{Overview}

Due to the advantages of regular monitoring, a plethora of glucometers are available in the market and most of them are based on biosensors. A biosensor is an analytical device that can integrate transform biological elements into electronic signals using a physiochemical transducer. The schematic diagram of biosensor is shown in Figure 2. In this device, a biological element such as analyte (glucose) will be detected by the transducer [12]. The transducer/detector then transforms the biological signals into electrical signals. The electronic circuit will process the signals and display the readouts in a user-friendly manner.

Over the past decades, home blood glucose monitoring (BGM) devices have provided a convenient method for the patient is to self-monitor their blood sugar levels without having to go to a clinic or hospital. Typically, the current method of blood glucose testing uses a small electronic device also knows as a glucometer. The meter reads the amount of sugar in a small sample of blood which is taken using a lancet usually from the fingertips and is placed on a chemically pre-treated disposable test strip. A reader is used to detect electrical signals from the chemical reactions on the test strip. While this method is convenient and relatively cheap, it is still only an instantaneous measurement of a patient's blood sugar level and causes 
minor discomfort due to the necessity of pricking the skin to draw blood. This method is still invasive and is unsuitable for continuous monitoring as multiple prickings carry the risk of infections and tissue damage in the long run.

Non-invasive approach can be classified as the approach where the patient would experience pain due to blood drawing procedures [13]. In a narrow definition, any medical approaches which does not involve the introduction of equipment or instrumentation item into the subject body is what is referred to as the non-invasive approach. There are quite numbers of research works developed for these non-invasive applications, including the near-field infrared spectroscopy, far-field infrared spectroscopy, etc. Several non-invasive techniques developed for glucose monitoring are reviewed in this section. The main technologies are electrochemical methods, microwave sensing, raman spectroscopy, near-infrared spectroscopy, iontophoresis and stepped-impedance resonator.

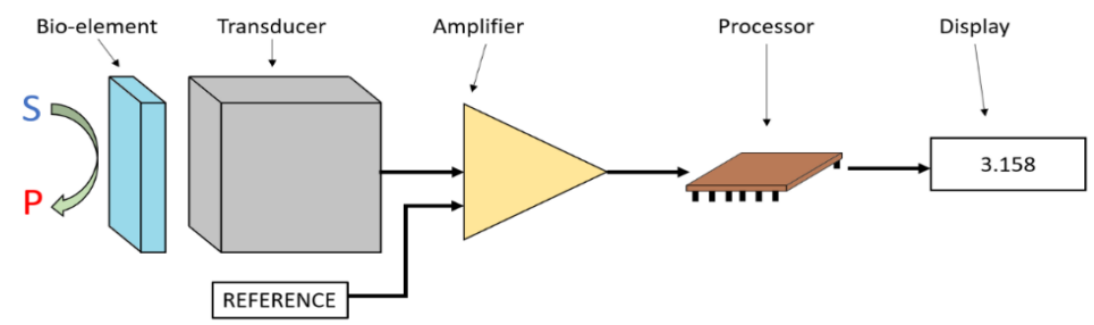

Figure 2. Building blocks of a biosensor

\subsection{Electrochemical methods}

Electrochemical technique has been used widely for glucose detection where it was developed to detect glucose levels in blood, tears [14, 15], saliva [16] and also sweat. Blood-based glucose monitoring have been used by the public since 1962, where the first generation of glucose biosensors used the electrochemical approach for glucose detection. Using this technique, indirect quantification of glucose concentrations was achieved by placing a thin layer of the glucose oxidase enzyme on a platinum electrode via a semipermeable dialysis membrane. This sensor measured the decrease in oxygen concentration and the liberation of hydrogen peroxide, which was proportional to the glucose concentration [11]. These glucose biosensors have evolved into the 'finger-pricking' approach that we have today, which uses very tiny blood samples which are tested in vitro using test strips and a glucometer. This enzymatic method measures snapshots of blood glucose levels, is invasive, inconvenient and painful. As a non-continuos method, it can overlook periods of hyper or hypoglycaemia which occurs outside of the sampling window.

An attempt to provide more continuous measurements has been done in which the glucometer is connected as a system to an app, iBGStar ${ }^{\circledR}$ Diabetes Manager. This app provides visual graphs and statistics to analyze glucose patterns on-the-go. To relieve users of the finger-pricking pain and inconvenience during blood glucose measurements, devices based on other biological fluids have been invented. Wilson and his team have developed tear-based electrochemical glucose sensing, which uses an electrodes-like hollow coil-formed with flexible wireless sensor system [14]. Claussen et al. developed an electrochemical biosensor to detect saliva and tear glucose using a complex photolithographic procedure involving graph nanosheets, platinum nanoparticles and the enzyme Glucose Oxidase [15]. Another device based on electrochemical sensing with carbon nanotubes uses saliva, has been developed by Wang et al. [16]. Considering the ease of access of the fluids, it is expected that there will be more research on devices using non-invasive biological fluids for glucose measurements in the near future.

\subsection{Microwave sensing of blood glucose levels}

Electromagnetic waves can be used to characterize permittivities of different materials using RF or microwave sensors. Dielectric properties of biological tissues have been extensively studied by researchers [19]. Recently, the wideband dielectric properties of biological tissues with different glucose concentrations have been studied and modeled using the single-pole Cole-Cole Model [20]. The Cole-ColeModel provides an effective and precise biological tissue representation over very wide range of frequencies and has recently been used to decrease the complexity of experimental data for different human tissues such as skin, bone, fat, brain and breast. The expression of single-pole is:

$$
\hat{\varepsilon}=\varepsilon_{\infty}+\frac{\varepsilon_{S}-\varepsilon_{\infty}}{1+(j \omega \tau)^{(1-\alpha)}}+\frac{\sigma_{i}}{j \omega \varepsilon_{0}}
$$


where $\varepsilon_{\infty}$ is the high frequency permittivity, $\varepsilon_{s}$ is the static permittivity, $\tau$ is the relaxation time constant, $\alpha$ is parameter that allows broadening of the dispersion and $\sigma_{i}$ is the onductivity of the material. The Cole-Cole model is modified via curve fitting to analyze the relationship between glucose concentrations and permittivity as well as conductivity in Gigahertz frequency regions. It has reported that resonant frequencies shift with respect to different concentrations of glucose.

Using the same concept of detecting dielectric permittivities, inductive and capacitive sensors can also be used to detect glucose [19]. Figure 3(a) shows the basic concept of the sensor, where the sensor structure is a flexible inductor that consists of the core and the coil, and the inductor's stray capacitance will change in reaction with the body glucose, when the inductor is in contact with the body. Impedance measurements can be correlated with the change in blood glucose levels in the body. In another device, two inductors are placed to sandwich the skin and tissue, and magnetic coupling between the two inductors can be measured using voltage or current [20]. The magnetic coupling not only depends on the media dielectric characteristics found between two coils, it is also proportional to the analyte type and concentrations. The ratio of glucose concentration is proportional to input and output voltages, or between currents. The input signal frequency plays a big role in producing enough coupling, and usually high frequencies such as $2.4 \mathrm{MHz}, 2.9 \mathrm{MHz}$ [21] or $7.7 \mathrm{GHz}$ are used [22].

Another device capable of measuring dielectric permittivities is the stepped impedance resonators (SIRs) as shown in Figure 3(b). SIRs are microwave-resonator-based biosensors that can determine the concentration or physical characteristics of a material under test based on the interaction between the resonator electromagnetic (EM) waves and the material [23]. Similar to the inductive capacitive sensors, the interaction of the electromagnetic waves with the material under test is reflected in the resonator's S-parameters as well as the shift of its central frequency [23]. The resonant frequency shift correlates with the physical characteristics of the material under test. Unlike the inductive capacitive sensor, the SIR is planar and can be fabricated using printed circuit boards. This gives it an advantage in terms of reduction of circuit size and possible integration in wearables. The SIR shown in Figure 3(b) is composed of alternating narrow and wide signal line sections where the narrow sections of the line. These cross-coupled SIRs can be used to detect changes in dielectric permittivities of glucose samples using $S_{11}$ measurements. Variations in the glucose concentration effectively change the equivalent series inductance and shunt capacitance of the biosensor resonator.

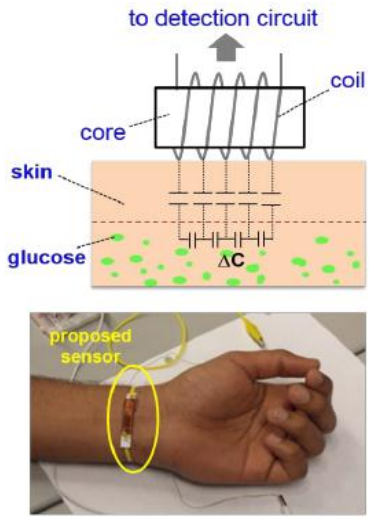

(a)

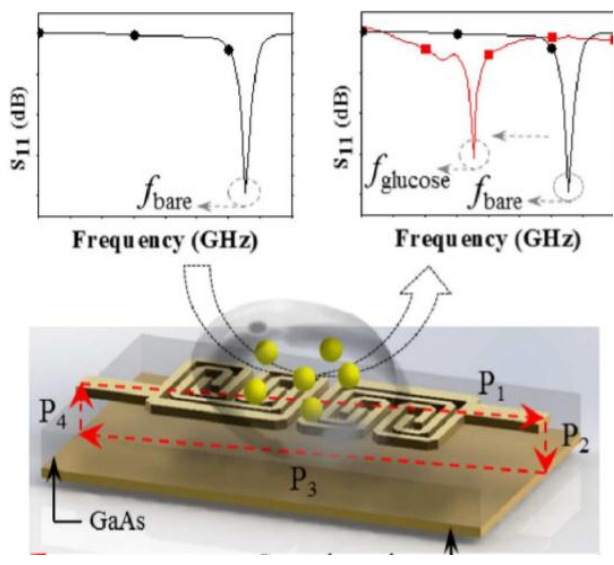

(b)

Figure 3. Microwave sensing, (a) An inductive capacitive sensor and its equivalent circuit model [21], (b) A stepped-impedance resonator biosensor for measurements of glucose levels [28]

\subsection{Near-infrared spectroscopy}

Near-infrared spectroscopy (NIRS) can detect various chemical components of biological specimens by measuring the absorbance of light by organic molecules. This method interrogates tissue using light waves and the reflected spectra from wavelengths between 400 and $2400 \mathrm{~nm}$ enables analysis of the specimen. Radiation in the NIR range can penetrate into skin deeper than visible radiation or MIR radiation [24]. The spectral region of NIR has some windows in which hemoglobin, water and lipid absorption band intensities are low enough to let the light penetrate into tissue which enable the spectral measurements of near-infrared. NIR measurements involves a light source(transmitter) and a light detector (receiver) placed on both sides of a soft tissue such as ear lobe. Ear lobes are 
commonly chosen because of its relatively small thickness and boneless muscle. NIR light is applied at the transmitter part, and the light will be attenuated due to the absorption from glucose in the blood. The attenuated signal will be processed at the receiver end of the system. However, the downside of using this technique is scattering of light inside the tissue that will cause interference from other types of molecules with similar absorption properties. Therefore, the device usually requires calibration of glucose value so that it could be calibrated to the actual patient glucose amount in the blood [25].

\subsection{Optical methods}

Surface plasmon resonance (SPR) is a concept that occurs when polarized light hits a thin layer of conductive and chemically inert metal electrode such as gold, interfacing with the media with different refractive index. In basic system of Kretschmann configuration, the light beam is focused onto the metal through a prism, forming a resonating surface plasmon polaritons (SPP) that are sensitive to the changes of glucose levels at certain resonance angle, $\theta \mathrm{R}$. Photons will be absorbed during this process, resulting in shifting of the dark line in the reflected light due to the variations of the glucose level which will be detected by the sensor $[33,35]$. Recent trends are focusing on enhancing the sensitivity by surface modification as can be seen in [36, 37]. Fluorescence technique is based on the principle of Stokes shift. In this principle, a wavelength difference is created after the absorption of radiation of a different energy level; emitting a specific fluorescent light in the process. A specialized molecule called fluorophores will be bound to the glucose molecule and emit fluorescent light proportional to the concentration of glucose presence in the sample analyte [28]. However, since it requires interaction of the fluorophores and glucose molecule, most of the application will requires physical samples such as blood [38], tears and saliva [39] and transdermal glucose from buffer solution [40]. Summaries of key approaches in noninvasive detection of glucose are indicated as in Table 3.

Table 3. Advantages and disadvantages of non-invasive glucose detection

\begin{tabular}{|c|c|c|}
\hline Glucose Detection & Advantage & Disadvantage \\
\hline $\begin{array}{c}\text { Electrochemical } \\
{[14-17]}\end{array}$ & $\begin{array}{ll}- & \text { Low power consumption } \\
- & \text { Wide linear range of sensor } \\
\text { response } \\
\text { - } \quad \text { Excellent repeatability and } \\
\text { accuracy }\end{array}$ & 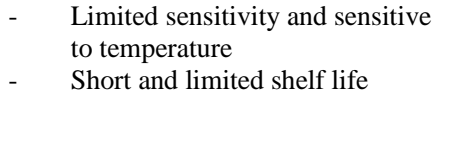 \\
\hline $\begin{array}{c}\text { Microwave Sensing } \\
{[20-23]}\end{array}$ & $\begin{array}{l}\text { - Detect rapid change in glucose } \\
\text { concentration } \\
\text { - Deep penetrate signal through } \\
\text { tissue } \\
\text { - } \quad \begin{array}{l}\text { Highly stable instrument } \\
\text { calibration }\end{array}\end{array}$ & $\begin{array}{ll}\text { - } & \text { Poor selectivity } \\
\text { - } & \text { Susceptible to biological differences } \\
& \text { in blood }\end{array}$ \\
\hline $\begin{array}{c}\text { Near-Infrared Spectroscopy } \\
{[30][31][32]}\end{array}$ & $\begin{array}{l}\text { - Minimum sample preparation } \\
\text { required } \\
\text { - } \quad \text { Measurement of blood glucose } \\
\text { in less than a minute. } \\
\text { - } \quad \text { Portable, compact and low } \\
\text { cost }\end{array}$ & $\begin{array}{l}\text { - } \quad \text { High scattering pattern } \\
\text { - } \quad \text { Selectivity problem to determine } \\
\text { the glucose }\end{array}$ \\
\hline $\begin{array}{c}\text { Iontophoresis } \\
{[34]}\end{array}$ & $\begin{array}{l}\text { - } \quad \text { Concentrated in a specific area } \\
\text { Glucose measurement is based } \\
\text { on the well-known enzymatic } \\
\text { method }\end{array}$ & $\begin{array}{ll}- & \text { Slight risk of electrode burns } \\
- & \text { Susceptible to sweating } \\
- & \text { Causes skin irritations }\end{array}$ \\
\hline $\begin{array}{c}\text { Optical methods } \\
{[28][33,35][38][39][40]}\end{array}$ & $\begin{array}{ll}\text { - } & \text { High resolution } \\
\text { - } & \text { Good sensitivity, selectivity } \\
\text { and reproducibility }\end{array}$ & $\begin{array}{ll}\text { - } & \text { Sensitive to motion and change in } \\
\text { temperature } \\
\text { - } \quad \text { Time consuming and lagging }\end{array}$ \\
\hline
\end{tabular}

\subsection{Commercial remote glucose sensors}

GlucoWatch is a wearable device which has been developed and introduce to the market as a non-invasive glucose monitoring by a person continuously as shown in Figure 4(a). The device which can detect the trends of glucose level among diabetic patients has been approved as a complementary to the conventional blood glucose meters in tracking the diabetic patterns. This device implements the iontophoresis technique. This technique extracts interstitial fluid to the skin's surface to measure the levels of glucose in range of $\mathrm{pH}$ 7.2-7.4. The entire process is generated when electric current of $300 \mu \mathrm{A}$ supplied between two electrodes that contact the skin at the back of device. The electric current allows the interstitial fluid to be taken out and brought into two collection disks which act as cathode and anode. Even though GlucoWatch has been considered as continuous and non-invasive device of glucose monitoring, this approach has been found to be restricted due to its necessity of periodic recalibration using pricking 
method. Thus it leads to increase in costs in patient care and testing equipment. Moreover, the glucose detections are affected by the skin temperature as well as the fluctuations of perspiration through applying both conductivity sensors and thermos transducers in the device. What makes it interesting is that this device able to measure up to six glucose readings per hour. It is also equipped with alarm to warn the level of glucose when the change is greater than $35 \%$ from borderline. The detection of glucose by GlucoWatch lags the standard enzymatic measurement methods by 15 minutes in time. Other disadvantages of GlucoWatch includes long warm up times, irritating skin rash, sweating which caused the removal of product in 2008 from market.

GlucoTrackTM which has been developed by Integrity Applications Ltd, acts as real-time, non-invasive glucose monitoring device as shown in Figure 4(b). The device able to detect the concentration of blood glucose through the employment of three techniques on non-invasive monitoring technique of glucose that known as electromagnetic, ultrasonic and heat capacity. GlucoTrackTM possess very high accuracy and precision due to combination of multiple technologies. It also able to reduce the noises in every measurement through minimization of interferences effect. The method of measuring blood glucose concentration is through the attachment of device that has personal ear clip (PEC) in the earlobe. PEC has been equipped with both sensors and calibration electronics. Earlobe which is an easiest accessible site due to an abundant supply of blood to it and it does not affect any routine activities of individual when the device is placed on it. Moreover, the device requires calibration individually which against invasive post-prandial and basal references of blood glucose prior to its application in glucose measurements. However, the calibration only valid for one month. It has high accuracy in clinical trials as the results is tally with other glucose analyzer and glucose meter available in the market. The device features include batter recharge, IR and USB connectivity, multiuser support, alerts sensor of hypo- and hyperglycaemia, wide LCD screen, internal memory to store up until 1000 readings per user, as well as available software to be used for data processing and analysis. The advantage is that the device is very lightweight, compact and safe for patients yet it still not commercialized as the supplier wanted to improves its calibration procedure quality and enhance its performance in data processing by improving its algorithm functions.

Abbott FreeStyle ${ }^{\circledR}$ Libre received CE Mark in 3 Sept 2014 and is used in seven European countries. The FreeStyle device does not need any finger pricks to check and calibrate. Individual only need to wear it at the back of the upper arm as shown in Figure 4(c) around 14 days. The measurement of blood glucose is done every minute in interstitial fluid using a needle that is $5 \mathrm{~mm}$ long and $0.4 \mathrm{~mm}$ wide which was inserted by the device under the skin using applicator held with adhesive tape. However, the application of finger stick test by blood glucose meter is needed when the blood glucose level is rapidly change as the levels of interstitial fluid glucose does not reflects accurately the level of blood glucose or whenever hypoglycaemia or impending hypoglycaemia is reported by the system. It also essential when the symptoms does not match the readings of system. The device needs up to one hour to equilibrate before it can obtain the glucose result in not more than one second. The device reader holds up to 90 days of data. Every scan will shows a current glucose reading, as well as 8-hour of glucose level history and the prediction of direction glucose is heading.

Medtronic Guardian ${ }^{\mathrm{TM}}$ Sensor is a sensor for continuous glucose monitoring that need to be placed at the back of individual's arm or on abdomen as shown in Figure 4(d). The device has received FDA approval as the first hybrid closed loop (HCL) for insulin delivery system in the world. Guardian ${ }^{\mathrm{TM}}$ Sensor can be worn until 7 days continuously by individual. The device is equipped with Change Sensor alert assist it is activated when the sensor does not perform well. The device sensors box includes 30 days of sensor wear to ensure the diabetes patients provided with safe, accurate and reliable sensor glucose. The device is linked with software and application that gains information from Medtronic diabetes management system directly before it generates reports that can be used during the doctor's consultation and for doctor to monitor their patients' progress.

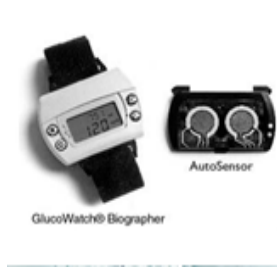

(a)

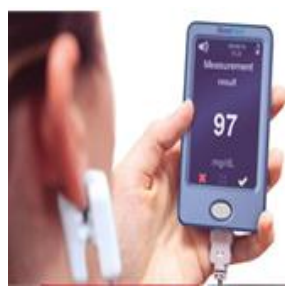

(b)

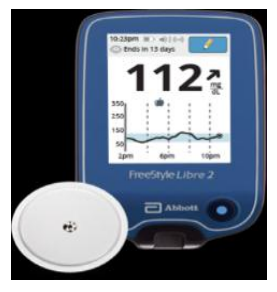

(c)

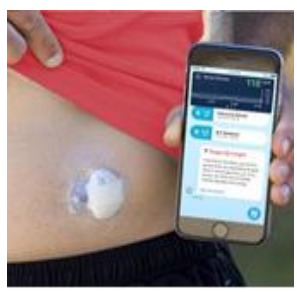

(d)

Figure 4. GlucoTrackTM, (a) GlucoWatch device is easily wearable on the wrists like a watch [41], (b) GlucoTrack non-invasive glucose monitoring device with personal ear clip [42], (c) FreeStyle Libre Flash Glucose Monitoring System that is placed at the back of upper arm [43], (d) Medtronic Guardian ${ }^{\mathrm{TM}}$ Sensor Glucose Monitoring System worn on either abdomen or back of upper arm [44] 


\section{CONCLUSION}

Diabetes is increasing worldwide as it effects about 7.8 million persons that developing diabetes each year. The frequent monitoring of glucose is the most important part of diabetic management as it is the only way by which the diabetics patient can keep their blood glucose level within the normal range. This will enable them to live a healthy life by avoiding diabetes-associated life-threatening complications. Several research efforts have been conducted to find the precise measurement sensor with easy way to monitoring the blood glucose. But it will take a lot of time based on the challenges involved and only a very limited scope for success. The painless non-invasive glucose monitoring will enable the diabetics to monitor their blood glucose more frequently. However, despite continuous developments in the technology for non-invasive glucose monitoring, more intensive research efforts are still required to develop a non-invasive glucose monitoring device for highly precise glucose measurements. In this work, various type of approaches for glucose sensing such as electrochemical biosensors (enzymatic, hexokinase), optical biosensors (surface plasmon resonance, fluorescence) and electrical biosensors (electromagnetic sensing, bioimpedance spectroscopy, reverse iontophoresis) have been described. Wearable sensors have the potential to play a major role in the continuous and non-invasive monitoring of biomarkers for diabetes and other disease conditions. Existing non-invasive glucose monitoring device such as Medtronic Guardian ${ }^{\mathrm{TM}}$ Sensor and Abbott FreeStyle ${ }^{\circledR}$ Libre, which already in the consumer technology market, provide an information base that can be expanded to include disease monitoring or diagnosis, and provide a tangible impact on health and wellness.

\section{ACKNOWLEDGEMENTS}

This work is supported by Malaysia Ministry of Higher Education (MOHE) under the Fundamental Research Grant Scheme (FRGS) FRGS17-030-0596.

\section{REFERENCES}

[1] American Diabetes Association, "Diagnosis and classification of diabetes mellitus," Diabetes care, vol. 37, pp. S81-S90, 2014.

[2] S. Coster, M. Gulliford, P. Seed, J. Powrie, and R. Swaminathan, "Monitoring blood glucose control in diabetes mellitus: a systematic review," Health technology assessment (Winchester, England), vol. 4, pp. i-iv, 1-93, 2000.

[3] G. Forte, B. Bocca, A. Peruzzu, F. Tolu, Y. Asara, C. Farace, et al., "Blood metals concentration in type 1 and type 2 diabetics," Biological trace element research, vol. 156, no. 1-3, pp. 79-90, 2013.

[4] P. Makaram, D. Owens, and J. Aceros, "Trends in nanomaterial-based non-invasive diabetes sensing technologies," Diagnostics, vol. 4, no. 2, pp. 27-46, 2014.

[5] E.-H. Yoo and S.-Y. Lee, "Glucose biosensors: an overview of use in clinical practice," Sensors, vol. 10, no. 5, pp. 4558-4576, 2010.

[6] D. H. Wasserman, "Four grams of glucose," American Journal of Physiology-Endocrinology and Metabolism, vol. 296, no. 1, pp. E11-E21, 2009.

[7] J. Vrba and D. Vrba, "A Microwave Metamaterial Inspired Sensor for Non-Invasive Blood Glucose Monitoring," Radioengineering, vol. 24, no. 4, pp. 877-884, 2015.

[8] M. E. Daly, C. Vale, M. Walker, A. Littlefield, K. G. Alberti, and J. C. Mathers, "Acute effects on insulin sensitivity and diurnal metabolic profiles of a high-sucrose compared with a high-starch diet," The American journal of clinical nutrition, vol. 67, pp. 1186-1196, 1998.

[9] B. A. Katchman, M. Zhu, J. Blain Christen, and K. S. Anderson, "Eccrine sweat as a biofluid for profiling immune biomarkers," PROTEOMICS-Clinical Applications, vol. 12, no. 6, p. 1800010, 2018.

[10] A. Asokan Thulasi, D. Bhatia, P. T. Balsara and S. Prasad, "Portable impedance measurement device for sweat based glucose detection," 2017 IEEE 14th International Conference on Wearable and Implantable Body Sensor Networks (BSN), Eindhoven, pp. 63-66, 2017.

[11] D. Bruen, C. Delaney, L. Florea, and D. Diamond, "Glucose sensing for diabetes monitoring: recent developments," Sensors, vol. 17, no. 8, pp. 1866, 2017.

[12] P. Mehrotra, "Biosensors and their applications-A review," Journal of oral biology and craniofacial research, vol. 6, no. 2, pp. 153-159, 2016.

[13] J. L. Smith, "The pursuit of noninvasive glucose: hunting the deceitful turkey," Revised and Expanded, copyright, 2015.

[14] H. Hanssen and R. Tweehuysen, "Electrochemical Biosensor Based on Hollow Coils, Method for Making and Use of the Sensor and a Medical Device Comprising the Sensor," ed: EP2699690B1, 2014.

[15] J. C. Claussen, A. Kumar, D. B. Jaroch, M. H. Khawaja, A. B. Hibbard, D. M. Porterfield, et al., "Nanostructuring platinum nanoparticles on multilayered graphene petal nanosheets for electrochemical biosensing," Advanced Functional Materials, vol. 22, pp. 3399-3405, 2012.

[16] W. Zhang and M. L. Wang, "Saliva Glucose Monitoring System," ed: Google Patents, 2016.

[17] R. Batool, A. Rhouati, M. H. Nawaz, A. Hayat, and J. L. Marty, "A Review of the Construction of Nano-hybrids for Electrochemical Biosensing of Glucose," Biosensors, vol. 9, no. 1, pp. 46, 2019. 
[18] R. Ahmad, M. Khan, M. R. Khan, N. Tripathy, M. I. R. Khan, P. Mishra, et al., "Nano-donuts shaped nickel oxide nanostructures for sensitive non-enzymatic electrochemical detection of glucose," Microsystem Technologies, pp. 1-6, 2020.

[19] E. Topsakal, T. Karacolak and E. C. Moreland, "Glucose-dependent dielectric properties of blood plasma," 2011 XXXth URSI General Assembly and Scientific Symposium, Istanbul, pp. 1-4, 2011.

[20] T. Yilmaz, R. Foster, and Y. Hao, "Radio-frequency and microwave techniques for non-invasive measurement of blood glucose levels," Diagnostics, vol. 9, p. 6, 2019.

[21] S. P. Cheggoju, "Development of Non-Invasive Glucose Sensor," University of Akron, 2016.

[22] M. F. A. bin Yunos, A. N. Nordin, A. Zainuddin, and S. Khan, "Modeling and development of radio frequency planar interdigital electrode sensors," Bulletin of Electrical Engineering and Informatics, vol. 8, no. 3, pp. 978-984, 2019.

[23] M. Jayakrishnan, S. Skaria, D. Sathyanath, and P. Mohanan, "RF assisted non invasive method for blood glucose monitoringa feasiblity study," in 2015 IEEE Applied Electromagnetics Conference (AEMC), Guwahati, 2015, pp. 1-2.

[24] A. Tura, S. Sbrignadello, D. Cianciavicchia, G. Pacini, and P. Ravazzani, "A low frequency electromagnetic sensor for indirect measurement of glucose concentration: in vitro experiments in different conductive solutions," Sensors, vol. 10, no. 6, pp. 5346-5358, 2010.

[25] C.-F. So, K.-S. Choi, T. K. Wong, and J. W. Chung, "Recent advances in noninvasive glucose monitoring," Medical Devices (Auckland, NZ), vol. 5, pp. 45-52, 2012.

[26] H. Melikyan, E. Danielyan, S. Kim, J. Kim, A. Babajanyan, J. Lee, et al., "Non-invasive in vitro sensing of dglucose in pig blood," Medical engineering \& physics, vol. 34, no. 3, pp. 299-304, 2012.

[27] R. A. Alahnomi, Z. Zakaria, Z. M. Yussof, T. Sutikno, H. Sariera, and A. A. M. Bahar, "Accurate characterizations of material using microwave T-resonator for solid sensing applications," Telecommunication, Computing, Electronics and Control, vol. 18, no. 1, pp. 99-105, 2020.

[28] K. K. Adhikari and N.-Y. Kim, "Ultrahigh-sensitivity mediator-free biosensor based on a microfabricated microwave resonator for the detection of micromolar glucose concentrations," IEEE Transactions on Microwave Theory and Techniques, vol. 64, pp. 319-327, 2015.

[29] A. Caduff, F. Dewarrat, M. Talary, G. Stalder, L. Heinemann, and Y. Feldman, "Non-invasive glucose monitoring in patients with diabetes: A novel system based on impedance spectroscopy," Biosensors and Bioelectronics, vol. 22, no. 5, pp. 598-604, 2006.

[30] J. Yadav, A. Rani, V. Singh and B. M. Murari, "Near-infrared LED based non-invasive blood glucose sensor," 2014 International Conference on Signal Processing and Integrated Networks (SPIN), Noida, 2014, pp. 591-594.

[31] M. A. Al-Dhaheri, N.-E. Mekkakia-Maaza, H. Mouhadjer, and A. Lakhdari, "Noninvasive blood glucose monitoring system based on near-infrared method," International Journal of Electrical \& Computer Engineering, vol. 10, no. 22, pp. 1736-1746, 2020.

[32] K. Prawiroredjo and E. S. Julian, "Comparative study of $940 \mathrm{~nm}$ and $1450 \mathrm{~nm}$ near infrared sensor for glucose concentration monitoring," Telecommunication, Computing, Electronics and Control, vol. 17, no. 2, pp. 981-985, 2019.

[33] W. Villena Gonzales, A. T. Mobashsher, and A. Abbosh, "The progress of glucose monitoring-A review of invasive to minimally and non-invasive techniques, devices and sensors," Sensors, vol. 19, no. 4, pp. 800, 2019.

[34] A. J. Bandodkar, W. Jia, C. Yardımc1, X. Wang, J. Ramirez, and J. Wang, "Tattoo-based noninvasive glucose monitoring: a proof-of-concept study," Analytical chemistry, vol. 87, no. 1, pp. 394-398, 2015.

[35] V. Amendola, R. Pilot, M. Frasconi, O. M. Maragò, and M. A. Iatì, "Surface plasmon resonance in gold nanoparticles: a review," Journal of Physics: Condensed Matter, vol. 29, no. 30, pp. 203002, 2017.

[36] H. Yuan, W. Ji, S. Chu, S. Qian, F. Wang, J.-F. Masson, et al., "Fiber-optic surface plasmon resonance glucose sensor enhanced with phenylboronic acid modified Au nanoparticles," Biosensors and Bioelectronics, vol. 117, pp. 637-643, 2018.

[37] D. Li, J. Wu, P. Wu, Y. Lin, Y. Sun, R. Zhu, et al., "Affinity based glucose measurement using fiber optic surface plasmon resonance sensor with surface modification by borate polymer," Sensors and Actuators B: Chemical, vol. 213, pp. 295-304, 2015.

[38] J. Yuan, Y. Cen, X.-J. Kong, S. Wu, C.-L. Liu, R.-Q. Yu, et al., "MnO2-nanosheet-modified upconversion nanosystem for sensitive turn-on fluorescence detection of $\mathrm{H} 2 \mathrm{O} 2$ and glucose in blood," ACS applied materials \& interfaces, vol. 7, no. 15, pp. 10548-10555, 2015.

[39] S. Kanjananimmanont, X. Ge, K. Mupparapu, G. Rao, R. Potts, and L. Tolosa, "Passive diffusion of transdermal glucose: noninvasive glucose sensing using a fluorescent glucose binding protein," Journal of diabetes science and technology, vol. 8, no. 2, pp. 291-298, 2014.

[40] C. Liu, Y. Sheng, Y. Sun, J. Feng, S. Wang, J. Zhang, et al., "A glucose oxidase-coupled DNAzyme sensor for glucose detection in tears and saliva," Biosensors and Bioelectronics, vol. 70, pp. 455-461, 2015.

[41] Mendosa.com, "The GlucoWatch Biographer," 2007. [Online]. Available: http://www.mendosa.com/glucowatch.htm [Accessed: 01- July- 2020].

[42] Hindustantimes.com, "Heard this? Smart sensor attached to your ear helps you track diabetes," 2017. [Online]. Available: https://www.hindustantimes.com/health-and-fitness/heard-this-smart-sensor-attached-to-your-ear-helpsyou-track-diabetes/story-cWaFV6MrKNQIFa8KzEfAXO.html. [Accessed: 01- July- 2020].

[43] Freestylelibre.us, "Introducing the FreeStyle Libre 2 system," 2018. [Online]. Available: https://www.freestylelibre.us/system-overview/freestyle-libre-2.html. [Accessed: 01- July- 2020].

[44] Medtronicdiabetes.com, "Our Most Advanced Sensor, Guardian ${ }^{\mathrm{TM}}$ Sensor 3," 2020. [Online]. Available: https://www.medtronicdiabetes.com/products/guardian-sensor-3. [Accessed: 01- July- 2020]. 


\section{BIOGRAPHIES OF AUTHORS}

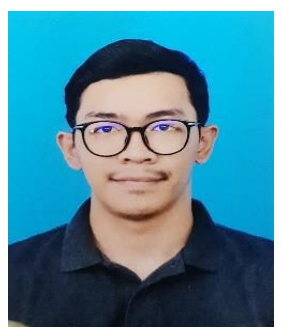

Muhammad Farhan Affendi Mohamad Yunos received his bachelor's degree from International Islamic University Malaysia, (IIUM) in 2017. Currently, he is a master's degree candidate at Department of Electronic and Computer Engineering, International Islamic University Malaysia. His research interest in microfluidics and modelling of MEMS devices.

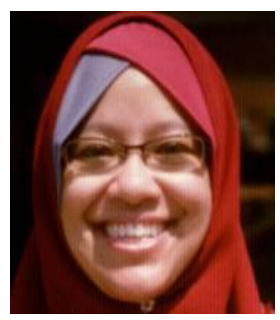

Anis Nurashikin Nordin has a bachelor's degree from International Islamic University Malaysia (IIUM) in 2000. She obtained both her master of science and doctor of science in computer engineering from the George Washington University, Washington D.C. in 2002 and 2008 respectively. Her field of specialization, both in her masters and doctorate degree is in microelectronics and VLSI. Currently, she is an associate professor in the Department of Electrical and Computer Engineering at the International Islamic University Malaysia, Kuala Lumpur. She is passionate about her research and her main research interests are in the area of biosensors, microfluidics, lab-on-chip devices, analog VLSI, RF-MEMS, and surface acoustic wave resonators. She has won several awards at the university level most recently best journal article award and best overall researcher award in 2018. She has also won numerous medals both internationally and locally for her research in developing personalized sensors for cancer chemotherapeutics. Among her awards is from the International European Women Inventors and Innovators Network Awards, Malaysian Technological Expo, British Invention Show, EU Special Inventor Award in the British Invention Show, International Trade Fair, Ideas, Inventions, New Products, Germany. 